\title{
Alarmierend hohe Rate saphenofemoraler Rezidive nach endovenöser Lasertherapie
}

\section{Alarmingly high rate of saphenofemoral recurrence following endovenous laser treatment}

Autoren

Achim Mumme, Dominik Mühlberger, Yazad Sidhwa, Thomas Hummel

Institute

Klinik für Gefäßchirurgie für Gefäßchirurgie,

Ruhr-Universität Bochum, Venenzentrum der

Dermatologischen und Gefäßchirurgischen Kliniken

Schlüsselwörter

Saphenofemorales Rezidiv, endovenöse Lasertherapie, Crossektomie und Stripping, Randomisierte kontrollierte Studien

Key words

sapheno femoral recurrence, endovenous laser ablation, crossectomy and stripping, randomised controlled trials

eingereicht 09.11 .2018

akzeptiert $\quad 09.11 .2018$

Bibliografie

DOI https://doi.org/10.1055/a-0798-6120

Phlebologie 2019; 48: 18-22

(c) Georg Thieme Verlag KG Stuttgart · New York ISSN 0939-978X

Korrespondenzadresse

Prof. Dr. med. Achim Mumme

Klinik für Gefäßchirurgie

Katholisches Klinikum der Ruhr-Universität Bochum,

Standort St. Josef-Hospital

Gudrunstr. 56

44791 Bochum

Tel.: 0234-509-2270

E-Mail: achim.mumme@ruhr-uni-bochum.de

\section{ZUSAMMENFASSUNC}

Bei den endovenösen Verfahren zur Behandlung der Stammveneninsuffizienz wird auf das Prinzip der Crossektomie verzichtet und es verbleibt nahezu regelhaft ein Crossenstumpf. Bei der operativen Therapie ist dagegen die Crossektomie ein unverzichtbarer Bestandteil des Behandlungskonzeptes und das Belassen eines Crossenstumpfes gilt als Behandlungsfehler, der die Ausbildung von Crossenrezidiven begünstigt. Im Bereich der Saphenacrosse gibt es daher momentan kein einheitliches Behandlungsprinzip. Was bei dem einen Verfahren als Therapieempfehlung gilt, ist bei dem anderen Verfahren ein Behandlungsfehler. Vor diesem Hintergrund sind Langzeitergebnisse der konkurrierenden Verfahrensweisen relevant. Insbesondere muss die Frage geklärt werden, ob die Abkehr vom Prinzip der Crossektomie tatsächlich ohne Auswirkungen auf die Rezidivrate ist.

In einer Literaturrecherche bei PubMed wurde nach prospektiven randomisierten Studien (RCT) mit mindestens 5 Jahren Nachbeobachtungszeitraum gesucht, in denen endovenöse Hitzeablationsverfahren mit der Crossektomie und StrippingOperation $(C+S)$ verglichen wurden und in denen die duplexsonographische Erfassung von Crossenrefluxen erfolgte. Das duplexsonographische Crossenrezidiv gilt als Surrogatparameter für das später auftretende klinische Crossenrezidiv. Es wurden insgesamt 7 RCTs identifiziert, in denen die o. g. Kriterien eingehalten wurden. In 6 RCTs wurde die Lasertherapie (EVLA) untersucht und in einer Studie die Radiowellentherapie. Zugunsten einer besseren Homogenität der Vergleichsgruppen wurde die einzige Radiowellenstudie exkludiert, so dass ausschließlich EVLA-RCTs eingeschlossen wurden. In keiner RCT war die Rate duplexsonographischer Crossenrezidive nach EVLA niedriger als nach C + S. In einer Studie waren die Rezidivraten gleich und in 5 Studien ergab sich ein Vorteil für die Operation. In diesen RCTs war die Rate duplexsonographischer Crossenrezidive nach EVLA 1,7-5,6mal höher als nach Operation. Die Datenlage in RCTs mit mittelfristigem Nachuntersuchungszeitraum deutet darauf hin, dass die Abkehr vom Prinzip der Crossektomie zumindest bei der EVLA zulasten einer höheren Rate von Crossenrezidiven geht. Angesichts der großen sozialmedizinischen Bedeutung des Crossenrezidivs sind diese Zahlen alarmierend, da mit hohen Folgekosten für die Behandlung der Rezidive gerechnet werden muss.

\section{ABSTRACT}

The principle of crossectomy is entirely dispensed with in endovenous procedures for the treatment of saphenous incompetence, and there, nearly always, persists a great saphenous vein stump. Conversely, the crossectomy is a crucial component of the treatment concept of operative therapy, i.e., crossectomy and stripping. The persistence of a great saphenous vein stump is considered a treatment error, which facilitates the development of a sapheno femoral recurrence. Thus, at the moment, 
there is no unifying treatment principle regarding the sapheno femoral junction. That which is recommended in one procedure is considered a lapse in another. In this situation, the long term results of competing procedures are relevant. In particular, the query to be addressed is, if indeed foregoing the principle of crossectomy is of no consequence to the rate of recurrence. We used the literature search on PubMed to look for prospective randomised controlled trials (RCT) with a minimum retrospective observation time frame of five years, in which endovenous thermal ablations were compared with crossectomy and stripping surgeries, and in which the duplex scan results regarding SFj reflux were compiled. An SFJ reflux on duplex scan is considered as a surrogate parameter for the later development of a clinical recurrence. In total, seven RCTs that adhered to the aforementioned criteria were found. Endovenous laser ablations (EVLA) were evaluated in six RCTs and radiofrequency ablations in one. For the benefit of a better uniformity of comparison groups, the sole radio frequency ablation study was excluded, so as, to only include the EVLA RCTs. SFJ reflux rate of EVLA was not lower than its surgical counterpart in any of the RCTs. The recurrence rate was equal in one study, and five studies yielded a significant advantage for the operative procedure. In these RCTs, the SFJ reflux rate post EVLA was 1.7-5.6 times higher than that post surgery. The data in the RCTs with intermediate follow-up time frames indicated that relinquishing the principle of crossectomy in EVLA leads to a higher rate of sapheno femoral recurrence. These results are alarming considering the socio-medical significance of recurrence, as the high follow-up financial cost for the treatment of recurrences has to be reckoned with.

\section{Einleitung}

Mit der Einführung der endovenösen Therapie Ende der 90er Jahre $[1,13]$ erfolgte ein Paradigmenwechsel. Das bis dahin geltende Prinzip der Crossektomie wurde verlassen und durch ein neues Behandlungskonzept ersetzt, bei dem nahezu regelhaft ein 1-2 cm langer Crossenstumpf unverschlossen bestehen bleibt. Bei der schon länger etablierten operativen Therapie ist allerdings bekannt, dass der Verzicht auf eine Crossektomie die Ausbildung von Crossenrezidiven begünstigt $[8,12]$. Inwieweit dieser Zusammenhang auch für die neue Therapieoption gilt, lässt sich nur anhand von kontrollierten Studien mit Randomisierung (RCT) klären.

Inzwischen verfügen wir über eine größere Anzahl von RCTs, die zur Klärung der Rezidivfrage dienen könnten [3,5,9,16,17,20]. Allerdings sind deren Follow-up-Intervalle mit bis zu 5 Jahren noch immer relativ kurz. Aus der deutschen Leistenrezidivstudie ist bekannt, dass die Reoperation von Crossenrezidiven im Durchschnitt erst ca. 14 Jahre nach dem Ersteingriff erfolgt [11]. Es dauert also wesentlich länger als 5 Jahre bis die Rezidive klinisch relevant werden und bis bei den Betroffenen ein erneuter Behandlungswunsch erwächst. Diese Zusammenhänge müssen bei der Beurteilung von Langzeitergebnissen der endovenösen Therapie berücksichtigt werden.

\section{Crossenrezidive kennzeichnen das Versagen der ursprünglichen Therapie}

Darüber hinaus wird die Beurteilung von Langzeitergebnissen erschwert durch die unvermeidliche Progredienz der Grunderkrankung. Neu aufgetretene Krampfadern ohne Verbindung zum eigentlichen Interventions-/Operationsgebiet können fälschlicherweise ein Versagen der Behandlungsmethode vortäuschen. Perrin et al. führten daher für die Beurteilung von Langzeitergebnissen die sogenannte REVAS-Klassifikation (Recurrent varices after surgery) ein, nach der zwischen Same-Site- und Different-Site-Rezidiven unterschieden wird [15]. Dabei sind die Different-Site-Rezidive gar keine Rezidive im eigentlichen Sinne. Es sind neu entstandene Varizen in den nicht vorbehandelten Gefäßprovinzen, die als Progredienz der Grunderkrankung zu gelten haben. Anders verhält es sich bei den Same-Site-Rezidiven. Diesen liegt ein Versagen der ursprünglichen Therapie zugrunde, das mit dem Wiederauftreten von initial behandelten Insuffizienzen einhergeht. Für die Rezidive nach Crossektomie und Stripping, bzw. nach endovenöser Therapie der Vena saphena magna bedeutet dies in aller Regel, dass die Crossenregion wieder zum proximalen Insuffizienzpunkt der Varikose wird ( $\triangleright$ Abb. 1).

Wenn es also um die Bestimmung von Langzeitergebnissen endovenöser oder operativer Therapieoptionen geht, sind in erster Linie die Crossenrezidive relevant. Es dauert allerdings lange, bis aus einem zurückgelassenen Stumpf ein klinisch relevantes Rezidiv hervorgeht. Erst nach einer Latenzphase von durchschnittlich 7-8 Jahren entwickeln sich bei operierten Patienten die ersten klinischen Anzeichen für ein Rezidiv [11]. Mit hoher Wahrscheinlichkeit gelten diese Zusammenhänge auch für die endovenöse Therapie. Die Beurteilung klinischer Rezidivzeichen macht daher innerhalb der Latenzphase des Crossenrezidivs wenig Sinn. Innerhalb der Latenzphase werden überwiegend nur die Different-Site-Rezidive erfasst, die sich dann in den Studienarmen mit annähernd gleicher Häufigkeit finden lassen. Alle bis dato vorhandenen RCTs befinden sich innerhalb der Latenzphase des Crossenrezidivs und erlauben daher keine validen Aussagen bezüglich der Same-Site-Rezidivraten unterschiedlicher Behandlungsmethoden.

\section{Surrogatparameter „duplexsonographisches Crossenrezidiv“"}

In dieser Situation kann aber auf sogenannte Surrogatparameter zurückgegriffen werden, die das letztendliche Outcome schon zu einem frühen Zeitpunkt anzeigen. Für die Beurteilung der Langzeitergebnisse von Behandlungsmethoden bei Stammveneninsuffizienz gilt der duplexsonographisch detektierbare Crossenreflux als Surrogatparameter [19]. Das sogenannte „duplexsonographische Crossenrezidiv“ geht nahezu regelhaft dem späteren klinischen Rezidiv voraus und kann daher schon in der Latenzphase die später zu erwartenden klinischen Rezidivzeichen ankündigen. Den Zusammenhang von duplexsonographisch detektierbaren Refluxen und der späteren Entwicklung variköser Konvolute konnten bereits Schultz-Ehrenburg et al. [18] in der Bochumer Venenstudie nachweisen. De Maeseneer et al. [2] bestätigten diese Beobachtung in 


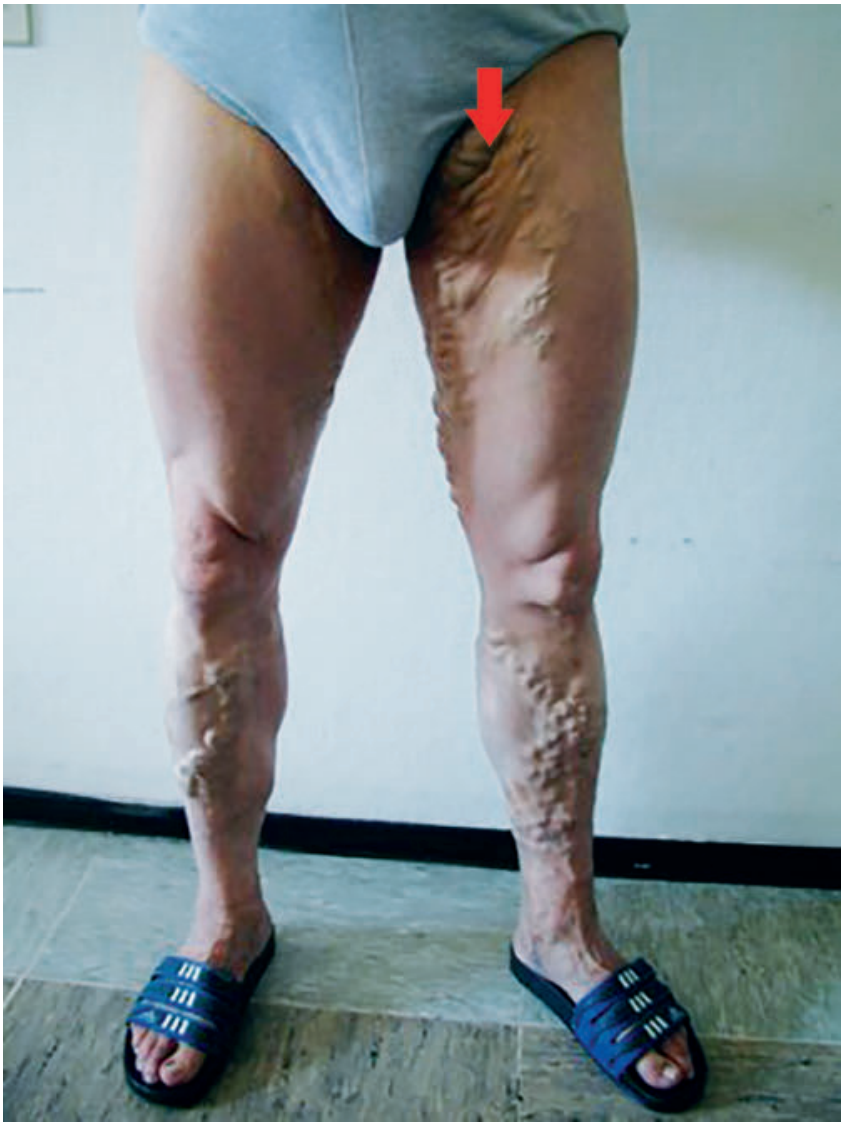

Abb. 1 Typisches Same-Site-Rezidiv. Crossektomie und Stripping vor ca. 15 Jahren. Seit ca. 7 Jahren zunehmende Rezidivvarikose ausgehend von einem lang belassenen Saphenastumpf.

einer prospektiven Studie. Anhand von duplexsonographisch festgestellten Crossenrefluxen konnte das spätere Auftreten klinischer Rezidive mit einer Sensitivität von $80 \%$ und einer Spezifität von $91 \%$ vorausgesagt werden.

\section{Methode}

In einer Literaturrecherche bei PubMed (August 2018) konnten 7 RCTs mit 5 Jahren Nachuntersuchungsintervall identifiziert werden, in denen die endovenöse Therapie mit der konventionellen Crossektomie und Stripping-Operation $(C+S)$ im Hinblick auf das duplexsonographische Crossenrezidiv verglichen wurde. Eine einzige Studie betraf die Radiowellentherapie. Aus Gründen der besseren Übersichtlichkeit wurde die Studie exkludiert. Diese Übersichtsarbeit beschränkt sich auf die Auswertung der verbliebenen 6 RCTs zur EVLA. Die Kriterien zur Bestimmung der duplexsonographischen Crossenrezidive waren in den verschiedenen Studien unterschiedlich, unzureichend oder gar nicht definiert, so dass auf eine Metaanalyse der Refluxraten verzichtet werden musste und nur ein direkter Vergleich der Ergebnisse innerhalb der jeweiligen Studien möglich war.
- Tab. 1 Duplexsonographisch detektierbare Crossenrezidive in prospektiven Studien mit Randomisierung und 5-Jahres-Follow-Up. Endovenöse Laserablation (EVLA) im Vergleich mit Crossektomie und Stripping $(C+S)$.

\begin{tabular}{|l|l|l|l|}
\hline & $\mathbf{n}$ & EVLA & $\mathbf{C + S}$ \\
\hline Rasmussen 2013 & $58 / 60$ & $8 \%$ & $4 \%$ \\
\hline Van der Velden 2015 & $78 / 69$ & $22 \%$ & $13 \%$ \\
\hline Rass 2015 & $185 / 161$ & $28 \%$ & $5 \%$ \\
\hline Flessenkämper 2016 & $142 / 159$ & $40 \%$ & $10 \%$ \\
\hline Gauw 2016 & $61 / 60$ & $49 \%$ & $23 \%$ \\
\hline Lawaetz 2017 & $144 / 142$ & $5 \%$ & $5 \%$ \\
\hline
\end{tabular}

\section{Ergebnisse}

In keiner Studie war die Rezidivrate nach EVLA niedriger als nach $\mathrm{C}+\mathrm{S}$. In einer Studie waren die Rezidivraten gleich und in 5 Studien ergab sich ein Vorteil für die Operation. In diesen Studien lagen die Rezidivrate in den EVLA-Studienarmen 1,7-5,6mal höher als in den operativen Vergleichskollektiven. Besonders deutlich waren die Unterschiede zwischen den Studienarmen in den deutschen RCTs. In der Studie von Rass et al. [17] war die Rezidivrate nach EVLA um den Faktor 5,6 höher, in der Studie von Flessenkämper et al. [3] um den Faktor 4.

\section{Diskussion}

Ein Großteil der in Deutschland durchgeführten Operationen dient zur Beseitigung von Crossenrezidiven. Nach den 2005 von Noppeney et al [14] veröffentlichen Daten eines deutschlandweiten Qualitätssicherungsregisters betrug der Anteil von Operationen bei Rezidiv ungefähr $15 \%$. In spezialisierten Zentren ist der Anteil von Re-Crossektomien sogar noch höher. Im Krangengut von Frings et al. [4] lag deren Anteil bei $24 \%$. Dementsprechend ist auch die sozioökonomische Bedeutung der Crossenrezidive hoch. Bereits im Jahr 2000 betrugen die Kosten deutschlandweit ca. 64 Mio $€$ [10]. Seinerzeit handelte es sich noch ausschließlich um „operative Rezidive“. Gegenwärtig erleben wir aber eine zunehmende Substitution der Varizenoperation durch endovenöse Verfahren. Diese Entwicklung dürfte zu erheblichen sozialmedizinischen Auswirkungen führen. Angesichts der in Deutschland um Faktor 4, bzw. 5,6 höheren Rezidivraten der EVLA drohen Folgekosten in astronomischer Höhe für die spätere Beseitigung von Crossenrezidiven. Diese betrügen, hochgerechnet mit den Zahlen aus dem Jahr 2000, ca. 256 Mio.-358,4 Mio. € pro Jahr, wenn statt der Operation nur noch EVLA zum Einsatz käme. Derartige Hochrechnungen sind sicherlich mit großen Unsicherheiten behaftet. Dennoch verdeutlichen sie, mit welchen Belastungen zu rechnen wäre, wenn die rezidivarme Operation durch endovenöse Methoden substituiert würde.

\section{Hohe Rezidivraten der Lasertherapie bestätigen die Notwendigkeit der Crossektomie}

Die hohen Rezidivraten der Lasertherapie sind als alarmierend anzusehen und werfen im Nachhinein die Frage auf, ob der bei Einführung der Hitzeablationsmethoden vorgenommene Paradig- 


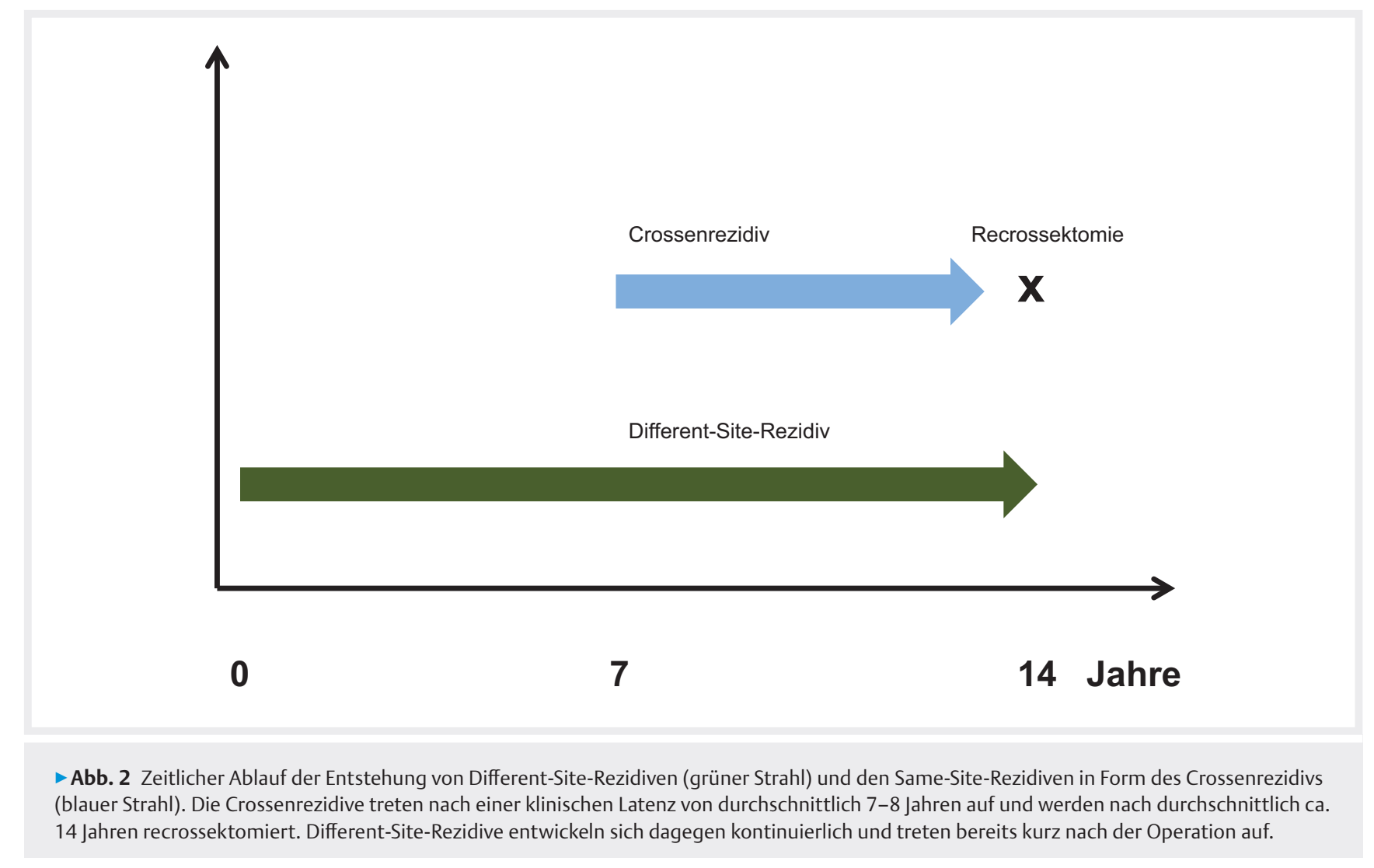

menwechsel berechtigt war. Die von der operativen Therapie her bekannten negativen Auswirkungen des Saphenastumpfes im Hinblick auf die Entwicklung von Rezidiven gelten offenbar auch dann, wenn der Stumpf als Folge einer endovenösen Behandlung entstanden ist. Demensprechend muss als Konsequenz für die endovenöse Therapie gelten, dass kein Crossenstumpf mit krankhaft veränderter V. saphena magna zurückgelassen werden darf. Diese Akzeptanz des Prinzips der Crossektomie würde schließlich auch das seit Jahren bestehende Paradoxon beenden, dass auf der einen Seite (endovenöse Therapie) erlaubt ist, was auf der anderen Seite (Operation) als Behandlungsfehler gilt und damit verboten ist. Tatsächlich bestätigten bislang zwei RCTs den positiven Effekt einer Kombination von EVLA und Crossektomie [3,7]. In Kombination mit einer operativen Crossektomie weist die Lasertherapie ähnliche Crossenrefluxraten auf wie die Crossektomie und Stripping-Operation.

\section{Ob das Prinzip der Crossektomie auch endovenös umgesetzt werden kann, ist fraglich}

Inwieweit allerdings das Prinzip der Crossektomie auf endovenösem Wege nachvollzogen werden kann, ist derzeit noch ungeklärt. Es fehlt ein Nachweis dafür, dass ein endovenöser Verschluss der Crosse im Niveau der tiefen Vene auch langfristig bestehen bleibt. Darüber hinaus ist ungeklärt, ob die Hitzeablation direkt an der tiefen Vene zu Folgeschäden wie Thromboembolie, narbigen Stenosen oder Schäden am lymphatischen Gewebe führt. Weitere Probleme bestehen in medicolegaler Hinsicht. Schließlich wird in den Anwendungshinweisen verschiedener Methoden die Hitzeablation der proximalen 1-2 cm der Stammvene ausdrücklich verboten. Bis zur Klärung dieser Fragen sollte die Hitzeablation direkt an der tiefen Vene nur innerhalb von ethisch abgesicherten Studien erfolgen.

\section{FAZIT}

Aus der Sicht der Patienten ist die Rezidivfreiheit ein wichtiger Faktor bei der Auswahl unterschiedlicher Therapieoptionen. Dementsprechend sollte beim Indikationsgespräch auf die gegenwärtige Studienlage hingewiesen werden. Patienten, denen die Rezidivfreiheit wichtig ist, sollte die Crossektomie und Stripping-Operation als Therapie der ersten Wahl angeboten werden. Bei den Nachkontrollen mit EVLA behandelter Patienten sollte dem hohen Risiko für die Entwicklung eines Crossenrezidivs Rechnung getragen werden, um ggf. mit einer Sklerosierungstherapie die Ausbildung eines klinisch relevanten Crossenrezidivs frühzeitig unterbinden zu können. Die weitere technische Entwicklung der Hitzeablationsverfahren sollte darauf abzielen, das Prinzip der Crossektomie auch auf endovenösem Wege umzusetzen.

\section{Interessenkonflikt}

Die Autoren geben an, dass kein Interessenkonflikt besteht. 


\section{Literatur}

[1] Boné C. Tratamiento endoluminal de las varices con laser de diodo. Estudio preliminary. Rev Patol Vasc 1999; 5: 35-46

[2] De Maeseneer MG, Vandenbroeck CP, Hendriks JM, et al. Accuracy of Duplex Evaluation One Year after Varicose Vein Surgery to Predict Recurrence at the Sapheno-Femoral Junction after Five Years. Eur J Vasc Endovasc Surg 2005; 29: 308-312

[3] Flessenkämper I, Hartmann M, Hartmann K et al. Endovenous laser ablation with and without high ligation compared to high ligation and stripping for treatment of great saphenous varicose veins: Results of a multicentre randomised controlled trial with up to 6 years follow-up. Phlebology. 2016; 31: 23-33

[4] Frings N, Glowacki P, Tran P et al. II. und III. Krossenrezidiv der Vena saphena magna/Vena saphena parva. Phlebologie 2006; 35: 1-6

[5] Gauw SA, Lawson JA, van Vlijmen-van Keulen C] et al. Five-year followup of a randomized, controlled trial comparing saphenofemoral ligation and stripping of the great saphenous vein with endovenous laser ablation (980 nm) using local tumescent anesthesia. J Vasc Surg 2016; 63: 420-408

[6] Hamann SAS, Giang J, De Maeseneer M et al. Five Year Results of Great Saphenous Vein Treatment: A Meta-analysis Eur J Vasc Endovasc Surg 2017; 54: 760-770

[7] Kalteis M, Adelsgruber P, Messie-Werndl S et al. Five-Year Results of a Randomized Controlled Trial Comparing High Ligation Combined With Endovenous Laser Ablation and Stripping of the Great Saphenous Vein. Dermatol Surg 2015; 41: 579-558

[8] Kluess HG, Noppeney T, Gerlach H et al. Leitlinie zur Diagnostik und Therapie des Krampfaderleidens. Im Internet: www.phlebology.de; Stand 15.10.2018

[9] Lawaetz M, Serup J, Lawaetz B et al. Comparison of endovenous ablation techniques, foam sclerotherapy and surgical stripping for great saphenous varicose veins. Extended 5 -year follow-up of a RCT. Int Angiol. 2017; 36: 281-288

[10] Mumme A, Olbrich S, Barbera L. et al. Saphenofemorales Leistenrezidiv nach Stripping der V. saphena magna: technischer Fehler oder Neovaskularisation? Phlebologie 2002; 31: 38-41.
[11] Mumme A, Burger P, Hummel T et al. Der lang belassene Saphenastumpf-Implikationen für die endovenöse Therapie der Varikosis. Phlebologie 2007; 36: 256-259

[12] Mumme A, Hummel T, Burger P. et al. Die Krossektomie ist erforderlich! - Ergebnisse der Deutschen Leistenrezidivstudie. Phlebologie 2009; 38: 99-102

[13] Navarro L, Min RJ Boné C. Endovenous laser: a new minimally invasive method of treatment for varicose veins - preliminary observations using an $810 \mathrm{~nm}$ diode laser. Dermatol Surg 2001; 27:117-122

[14] Noppeney T, Eckstein HH, Niedermeier H et al. Ergebnisse des Qualitätssicherungsprojektes Varizenchirurgie der Deutschen Gesellschaft für Gefäßchirurgie. Gefässchirurgie 2005; 10: 121-128

[15] Perrin MR, Guex JJ, Ruckley CV et al. Recurrent Varices after Surgery (REVAS), a Consensus Document. Cardiovascular Surgery 2000; 8 : 233-245

[16] Rasmussen L, Lawaetz M, Bjoern L et al. Randomized clinical trial comparing endovenous laser ablation and stripping of the great saphenous vein with clinical and duplex outcome after 5 years. J Vasc Surg 2013; 58: 421-426

[17] Rass K, Frings N, Glowacki P et al. Same Site Recurrence is More Frequent After Endovenous Laser Ablation Compared with High Ligation and Stripping of the Great Saphenous Vein: 5 year Results of a Randomized Clinical Trial (RELACS Study). Eur J Vasc Endovasc Surg 2015; 50: 648-656

[18] Schultz-Ehrenburg U, Reich-Schupke S, Robak-Pawelczyk B et al. Prospective epidemiological study on the beginning of varicose veins Bochum Study I-IV Phlebologie 2009; 38: 17-25

[19] Van den Bos R, Arends L, Kockaert M et al. Endovenous therapies of lower extremity varicosities: A meta-analysis J Vasc Surg 2009; 49: 230-239

[20] Van der Velden SK, Biemans AA, De Maeseneer MG. Five-year results of a randomized clinical trial of conventional surgery, endovenous laser ablation and ultrasound-guided foam sclerotherapy in patients with great saphenous varicose veins. Br J Surg. 2015; 102: 1184-1194 